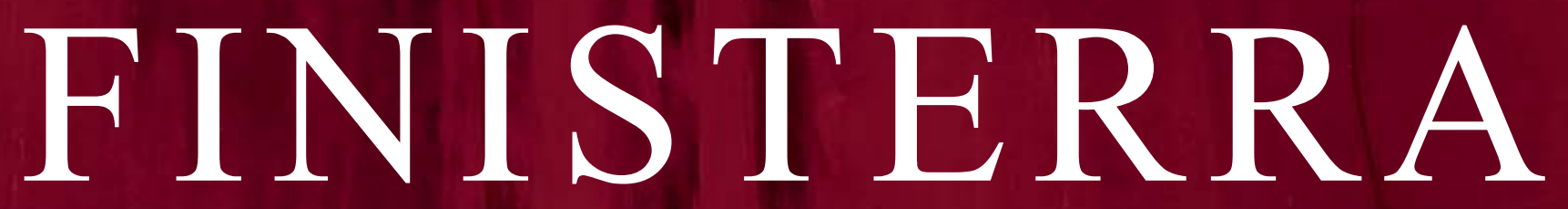

Revista Portuguesa de Geografia

Centro

de Estudos

Geográficos

Volume LII

Número 106

Dezembro 2017 


\title{
REFLEXIONES ACERCA DE LA DELIMITACIÓN Y DEFINICIÓN DEL MEDIO RURAL. DISEÑO DE UN ÍNDICE DE RURALIDAD PARA GALICIA ${ }^{i}$
}

\author{
Francisco Xosé Armas Quintá ${ }^{1}$ \\ Xosé Carlos Macía ArCE²
}

\begin{abstract}
RESUMEN - La tarea de delimitar y definir el medio rural ha sido, y sigue siendo, una misión compleja en la que la mayor parte de los investigadores sociales admiten que no existe una definición estricta y consensuada sobre el término. En el presente artículo se expone una revisión de la literatura científica acerca de las distintas conceptualizaciones del medio rural y sus posibles delimitaciones. Junto a ello se propone un índice de ruralidad para Galicia, una región del noroeste de España con un medio rural muy diverso fruto de las importantes transformaciones que han tenido lugar desde mediados del pasado siglo.
\end{abstract}

Palabras clave: Medio rural; índice de ruralidad; agricultura; pluriactividad; desarrollo.

RESUMO - REFLEXÕES ACERCA DA DELIMITAÇÃO E DEFINIÇÃO DO ESPAÇO RURAL. DESENHO DE UM ÍNDICE DE RURALIDADE PARA A GALIZA. A tarefa de delimitar e definir o espaço rural foi, e continua a ser, uma missão complexa na qual grande parte dos investigadores sociais admite não existir uma definição estrita e consensual sobre o termo. No presente artigo expõe-se uma revisão da literatura científica sobre as distintas conceptualizações do espaço rural e as suas possíveis delimitações. Adicionalmente, propõe-se um índice de ruralidade para a Galiza, uma região do noroeste de Espanha com um espaço rural muito diverso devido às importantes transformações que tiveram lugar desde meados do século passado.

Palavras-chave: Espaço rural; índice de ruralidade; agricultura; pluriatividade; desenvolvimento.

1 Profesor del Departamento de Didácticas Aplicadas, Universidad de Santiago de Compostela. Facultad de Formación del Profesorado, Avda. de Ramón Ferreiro, s/n., 27002, Lugo, España. E-mail: francisco.armas@usc.es

2 Profesor del Departamento de Didácticas Aplicadas, Universidad de Santiago de Compostela. Facultad de Ciencias de la Educación, Avda. de Xoán XXIII, s/n., 15782, Santiago de Compostela, España. E-mail: carlos.macia@usc.es 


\begin{abstract}
REFLECTIONS ON THE DELIMITATION AND DEFINITION OF RURAL AREAS. THE DESIGN OF A RURAL INDEX FOR GALICIA. The task of delimiting and defining rural areas has been, and continues to be, a complex one. Indeed, most social researchers admit to there being an absence of a strict, consensual definition of the term. In this article, a review of scientific literature looking at various conceptualisations of rural areas and their possible boundaries is discussed. Together with this, an Index of Rurality for Galicia is proposed, a region in north western Spain with highly diverse rural areas due to major transformations which have taken place since the middle of last century.
\end{abstract}

Keywords: Rural areas; index of rurality; agriculture; pluriactivity; development.

RÉSUMÉ - RÉFLEXIONS SUR LA DÉLIMITATION ET LA DÉFINITION DU MILIEU RURAL. ÉLABORATION D'UN INDICE DE RURALITÉ VALABLE POUR LA GALICE. Délimiter et définir le milieu rural a toujours été, et continue à être, une tâche complexe et la plupart des enquêteurs sociaux admettent qu'il n'existe pas de définition stricte et consensuelle de cette notion. On réalise ici une révision de la littérature scientifique concernant les divers concepts relatifs au milieu rural et à ses limites, et on propose un indice de ruralité valable pour la Galice, cette région du nord-ouest de l'Espagne dont le milieu rural, très divers, a subi des transformations importantes depuis la moitié du siècle passé.

Mots clés: Milieu rural; indice de ruralité; agriculture; pluriactivité; développement.

\title{
I. INTRODUCCIÓN
}

La definición y delimitación de las áreas rurales ha sido, y sigue siendo, una tarea compleja por las múltiples variables que deben ser analizadas y la gran diversidad de estos espacios. El concepto de lo rural ha pasado en las dos últimas décadas de ser sinónimo de tosco o atrasado, a tener connotaciones positivas y asociarlo a calidad (Sancho Comíns \& Reinoso Moreno, 2012). Campo y ciudad, rural o urbano, no tienen el mismo significado en cualquier parte del mundo. En algunos casos, se podría afirmar que ha llegado la urbanización del campo a muchos países desarrollados y la ruralización de las ciudades a otros en vías de desarrollo (García Ramón, Tlla i Pujol, \& Valdovinos Perdices, 1995). El medio rural es frecuentemente dotado de una importancia simbólica como indicador de la identidad nacional, o bien como oposición a la modernidad (Woods, 2011).

Las transformaciones que tuvieron lugar en el medio rural son, en la actualidad, un hecho incuestionable tanto a nivel espacial, funcional y socioeconómico (Sancho Comíns \& Reinoso Moreno, 2012) y han sido objeto de estudio de numerosas disciplinas interesadas en su análisis y evolución (Prados, 2009a). Lo rural se ha considerado, durante muchos años, sinónimo de agrario y aunque en la actualidad ese vínculo esté, en algunos casos muy presente, existe una gran variedad de usos y actividades económicas (García Ramón et al., 1995). Las áreas rurales han pasado de tener un papel básicamente agrario, de provisión de alimentos, materias primas y recursos humanos a las ciudades, hacia un 
perfil más pluriactivo y multifuncional (Rubio Terrado, 2010), siendo objeto de cambios estructurales a una escala sin precedentes como consecuencia de la transformación de su economía y sociedad (Shucksmith \& Chapman, 1998).

El medio rural actual muestra una complejidad de usos y funciones que se contrapone con la polarización claramente definida entre campo y ciudad de la primera mitad del siglo XX (García Ramón et al., 1995). Esta evolución hace que el medio rural comience a ser visto no como una simple superficie de producción, sino como un espacio que aporta una serie de valores de elevado interés para la sociedad global (paisaje, cultura, justicia social y alimentación saludable) y cada vez más urbanizada (Armesto López, 2005). Lo rural está presente en la imaginación popular y se revela tanto como un espacio imaginativo conectado con todas las clases de significados culturales, que van desde lo idílico hasta lo opresivo, como objeto de deseo de estilo de vida (Cloke, 2006). Estos espacios son, a menudo, valorados por sus paisajes escénicos y entornos naturales, pero también por ser el hogar de culturas indígenas diversas que atestiguan formas de vida tradicionales (Woods, 2011).

Las variadas funciones y significados que han sido atribuidos al medio rural, han hecho de lo rural un concepto complejo y ambiguo, siendo muy difícil tanto explicar por qué un espacio es rural o urbano, como trazar una línea en un mapa que separe a ambos (Woods, 2011). Ya no se puede hablar de una frontera de lo urbano y de lo rural sino de un "gradiente de urbanización" en lo que se refiere a la intensidad de la edificación, en la actividad y estructura económica, o en la movilidad de personas y mercancías (Roca, 2003).

Una de las debilidades de la literatura científica que aborda la definición del medio rural y su delimitación, es la asociación que se hace, en una parte importante de las investigaciones, de la agricultura con el medio rural (García Fernández, 1975; Bouhier, 1979; Villares Paz, 1982; Halfacree, 1993; Marsden, Murdoch, Lowe, Munton, \& Flynn, 1993). Hoy día es bien conocido el declive de la agricultura y el sector primario en su conjunto a favor de otras actividades como es el caso de los servicios (Shucksmith \& Chapman, 1998). Es cada vez más necesario intentar definir y delimitar las áreas rurales, no sólo para fijar el "nebuloso" concepto de lo rural sino para poder medir las diferencias en los distintos grados de ruralidad en cada territorio (Cloke, 1977) y adecuar así las líneas de actuación de las políticas orientadas a promover su desarrollo (López González, Crecente Maseda, \& Miranda Barros, 2004).

\section{LA PERSEVERANCIA EN LA DEFINICIÓN DEL MEDIO RURAL}

Las posturas acerca de la delimitación y definición de las áreas rurales son muy diversas dado que se trata de un proceso complejo asociado, en ocasiones, a un cierto matiz de subjetividad. Hay autores que relativizan la vigencia de la distinción rural-urbano (Camarero, 1993), aquellos que defienden la ruptura de lo rural con lo agrario (García Sanz, 1998), y los que consideran inútil o bien imposible, cualquier intento de delimitación conceptual del término rural (Bericat Alastuey, 1993; Sancho Hazak, 1997). La sociedad 
rural y el espacio rural ya no deben ser vistos como algo indivisible ya que la ruralidad está caracterizada por una multitud de espacios sociales que solapan una misma área geográfica. Mientras los espacios geográficos de la ciudad y el campo pueden llegar a difuminarse, no ocurre lo mismo con la distinción social de la ruralidad donde sus diferencias con la ciudad aún prevalecen (Cloke, 2006).

En el pasado, la emigración a las ciudades, el crecimiento urbano, y la concentración de la actividad económica definían a los centros urbanos, mientras que el medio rural perdía población y sufría el abandono y la marginalidad. Pero la situación actual es mucho más compleja y la disposición de factores no está tan polarizada debido a que la globalización establece un corredor de interrelaciones de las características demográficas, residenciales, sociales y económicas en las zonas urbanas y rurales (Prados, 2009a).

La definición de lo rural puede llegar a resultar confusa ya que las investigaciones sobre aspectos rurales multidisciplinares, parecen dar carta blanca para llevar a cabo muchos tipos de investigaciones con este título (Muilo, 2010). Existe la necesidad de delimitar las áreas rurales de una forma clara y multidimensional, dado que las primeras definiciones fueron tratadas como un concepto unidimensional, utilizando criterios en su delimitación basados casi todos en la variable demográfica, omitiendo factores tan notables como los socioculturales (Bosak \& Perlman, 1982). El hecho de que existan distintas percepciones sobre los elementos naturales, los económicos o los culturales que caracterizan la ruralidad, fue lo que llevó a la amplia diversidad de las definiciones propuestas (Entrena-Durán, 2012).

La dificultad de definir las áreas rurales llevó a una buena parte de los autores a intentar definir el medio rural en base a una división a tres niveles: áreas rurales remotas, áreas rurales centrales, y áreas rurales próximas a las centrales con diferentes grados de ruralidad. Esta distinción puede ser válida en espacios rurales dentro de un mismo país, pero no para llevar a cabo comparaciones a nivel internacional dada la gran diversidad entre ellas, tanto desde el punto de vista demográfico como en su estructura territorial (Muilo, 2010). A este respecto Cloke (2006) reconoce tres grandes marcos teóricos significativos en lo que se refiere a la construcción de la ruralidad. El primero de ellos puede asociarse a conceptos funcionales de la ruralidad, identificando elementos funcionales de la sociedad y el paisaje rural. El segundo está representado por el uso de conceptos político-económicos de cara a clarificar la naturaleza y posición de lo rural, reconociendo que las áreas rurales están conectadas con las dinámicas de la economía política nacionales e internacionales, y lo que ocurre en el medio rural está relacionado con lo que ocurre fuera de estas áreas. Por último, la tercera corriente teórica hace referencia a las construcciones sociales de la ruralidad.

Cada vez más, y sobre todo en medios rurales occidentales, tienen lugar multiplicidad de actividades y usos que pueden ser considerados urbanos. Por otra parte, también se han extendido al medio rural pautas de comportamiento y modos de vida urbanos, lo que provoca que, cada vez más, los límites entre lo rural y lo urbano sean más difusos y máxime en el contexto de la Sociedad de la Información y al proceso globalizador en el que vivimos (Esparcia, 2012). Para Halfacree (1993), la proliferación de representaciones 
diversas de los espacios rurales está llevando a que la imagen de lo rural se esté desprendiendo del espacio rural geográfico, es decir, la forma en el que el campo está siendo imaginado en los discursos populares quizá tenga poca correspondencia con las realidades actuales de los espacios rurales y la vida rural. Según Rubio Terrado (2010), cuando un medio rural no coincide con el modelo de ruralidad, nuestra percepción inicia un desplazamiento hacia la noción de lo urbano. Pero la diversidad creciente de las áreas rurales y la dificultad de establecer una delimitación llevó a Friedland (2002) a subrayar que ya no solo la ciudad es urbano ni campo lo rural. Prados (2009a) asiente que la expansión de los procesos de urbanización hacia zonas rurales periféricas es una consecuencia de la madurez de las metrópolis, y que el envejecimiento demográfico y la llegada de inmigración extranjera están presentes tanto en las ciudades como en el campo.

En la actualidad, las definiciones del medio rural engloban conjuntos muy heterogéneos con los consecuentes problemas e imprecisiones a la hora de su caracterización y delimitación. La preocupación por delimitar y definir el medio rural está motivada por la creciente importancia que adquieren las estadísticas oficiales para monitorizar las políticas públicas y la asignación de recursos (Sabalain, 2011). A este respecto Woods (2011) señala que la necesidad de los gobiernos de delimitar las áreas rurales para aplicar las políticas rurales, ha perpetuado el uso de definiciones cuantitativas del medio rural, dando lugar a una serie de definiciones poco congruentes. En esta línea Rodríguez (2011) apunta la necesidad de cambiar la forma de medir lo rural, pues debe haber una combinación de criterios con el fin de capturar la heterogeneidad de los espacios rurales.

Fueron muchos los autores que han intentado, con un éxito limitado, definir y categorizar el medio rural. Entre ellos se podrían citar a Cloke (1977), Hoggart (1990), Clout (1993), Cloke y Goodwin (1993), Halfacree (1993), Lázaro Araujo (1995), Ceña-Delgado (1995); García de León (1996); Guibertau Cabanillas (2002), Waldorf (2006), Sabalain (2011) y Rodríguez (2011) entre otros. Pese a haber muchas características que pueden ser consideradas a la hora de definir los espacios rurales, Ramos y Romero (1995) sostienen que estas áreas son, desde el punto de vista territorial, una fuente de recursos, receptor de residuos y soporte de actividad y Marsden et al. (1993), un almacén de pequeñas empresas emprendedoras que son vistas como un aspecto clave de dinamismo económico y de innovación.

Márquez Fernández (2002), asiente que el concepto de medio rural tiene, en la actualidad, diversas acepciones que están vinculadas al paisaje, al medio, al espacio, al territorio, al desarrollo, etc. y de ahí que las áreas rurales sean, por su variedad y heterogeneidad, muy difíciles de clasificar. La atracción que las áreas naturales protegidas ejercen sobre un determinado sector de población que desea vivir, trabajar y disfrutar de su tiempo libre en estos espacios, fue definido por Prados (2009b) con el concepto de naturbanización. Este concepto incluye el deseo de un nuevo medio ambiente residencial, la recuperación de actividades económicas tradicionales y aquellas que se basan en la tradición y el patrimonio cultural. La naturbanización tiene sus raíces en el concepto de contraurbanización y se asienta en procesos de desconcentración urbana y sus efectos en la distribución de la población en las áreas rurales (Prados, 2009b). 
Otros autores entienden diversas formas de comprender lo rural, una forma de hábitat en la que una población se adscribe a un lugar en el que el paso del tiempo forja una tradición, y una forma de ocupación en la que la actividad agraria ya no prevalece y se convierte en un espacio pluriactivo (García Sanz, 1998). Lo que sí parece incuestionable es que se debe hablar de pluralidad de áreas rurales que, además, comparten características comunes (De Arriba, 2011).

Lois González (2014) apunta otra forma de definir el medio rural teniendo en cuenta el nivel de artificialización del territorio y señala que, mientras en las ciudades es casi imposible observar ningún elemento del paisaje o de espacio originarios, en el rural la humanización de los lugares es moderada y con un conjunto de paisajes característicos. A este respecto Pratt (1996) señala que, aunque no sea posible precisar una "verdadera" ruralidad, quizás sea posible identificar algunos discursos que apoyen las desiguales relaciones sociales, escenarios económicos, etc. En el caso europeo, Ballas, Kalogeresis y Labrianidis (2003) señalan que las áreas rurales son extremadamente diversas y no pueden ser definidas únicamente en base a un simple criterio cuantitativo, siendo necesarias metodologías más sofisticadas que tengan en cuenta variables demográficas, económicas, sociales, etc.

\section{LO RURAL Y LO URBANO: ALGUNOS INTENTOS EN SU DELIMITACIÓN}

La dificultad para definir y delimitar las áreas rurales llevó a las administraciones públicas, a organismos nacionales e internacionales y a investigadores, a establecer criterios para concretar elementos y problemáticas que ayudasen a definirlas y delimitarlas, utilizando una mezcla de criterios objetivos y subjetivos (García Ramón et al., 1995).

Uno de los trabajos pioneros más relevantes al respecto fue el que llevó a cabo Cloke (1977) al diseñar un índice de ruralidad para Inglaterra y Gales. Clout (1993) diferenció para las áreas rurales europeas tres grandes grupos: espacios rurales dinámicos con crecimiento económico, espacios rurales con bajos niveles de desarrollo económico, pero con un gran potencial de futuro, y aquellos espacios rurales en proceso de abandono con densidades de población muy bajas y con recursos ambientales poco aptos para la agricultura. Del mismo modo que Clout (1993) y Ballas et al. (2003), presentaron una tipología de áreas rurales europeas a nivel regional utilizando técnicas de análisis estadístico de clústeres con el objetivo de agrupar las regiones europeas y definir así varios tipos de áreas rurales.

Halfacree (1995) intentó definir el medio rural a partir de representaciones sociales, y entre los aspectos que fueron atribuidos a las áreas rurales fueron aquellos relacionados con el medio ambiente, lo inexplotado, lo bello, el aire puro, etc. Este autor intenta aproximarse a la diversidad y variedad de vidas y experiencias que hoy día se encuentran en las áreas rurales, defendiendo que no se puede menospreciar aquello que no es ordinario en la vida rural. Insiste en la diversidad de las características sociales y económicas de la población que decide ir a vivir al rural, así como de experiencias 
de contraurbanización inusuales lejos de los patrones que habitualmente lo caracteriza, calificando a este proceso con el término de "contraurbanización olvidada" (Halfacree, 2007). A este respecto Lawrence (1996) sostiene que es importante reconocer el modo en el que el campo ejerce de un lugar común extraordinario donde la gente que vive allí se apoya en la creación de una variedad de relaciones entre la tecnología, las personas y la naturaleza.

Otros autores llevaron a cabo clasificaciones de las áreas rurales en sus respectivos países como es el caso de Márquez Fernández (2002). Esta autora, partiendo de la dificultad que ello implica, delimitó para el caso de España, tres tipos de zonas rurales poco favorecidas: áreas de montaña, espacios rurales con un elevado riesgo de despoblamiento, y áreas rurales donde es necesario que continúe la actividad agraria, pese a sus limitaciones, para la conservación del medio natural. También surgieron tentativas de desarrollo de índices simples como el que diseñó Esparcia (2012) conjugando diferentes indicadores. Este autor diferenció para la realidad valenciana dos grados de ruralidad en función del número de criterios que cumplía cada municipio.

Bibby y Shepherd (2004), distinguen tres aspectos que pueden ser utilizados a la hora de delimitar las áreas urbanas y rurales: la superficie construida, el particular sistema de asentamientos, y el tercer aspecto está relacionado con el término urbano el cual, ha sido usado para denotar una separación económica de la "tierra" como fuente directa de ingresos y generación de riqueza. La baja densidad de población, el elevado número de explotaciones agrícolas o la población dedicada al sector primario y la lejanía de los centros urbanos, son características que podrían identificarse y asociarse a las áreas rurales. Teniendo en cuenta este argumento, Waldorf (2006) diseñó un índice de ruralidad para los Estados Unidos partiendo de cuatro variables: tamaño demográfico, densidad de población, extensión del área urbana, y lejanía o perificidad.

Desde un punto de vista regional, López Ratón y Santiago Pérez (2005) elaboraron un índice de ruralidad para delimitar y clasificar los municipios gallegos a partir de varios indicadores demográficos y de ocupación. Se basaron, para la construcción del índice, en 15 indicadores de los que resultó un índice de ruralidad que oscila entre 0 y 1 , siendo los valores más próximos a cero los de mayor ruralidad. En su aplicación para el caso de Galicia, resultaron ser rurales 248 municipios (79\%), semiurbanos 60 (19\%) y urbanos 7 (2\%).

López González et al. (2004), ponen énfasis en la necesidad de delimitar el medio rural con el fin de orientar, de una forma más correcta, las líneas de actuación de las políticas de desarrollo rural. Para ello subrayan que la unidad mínima de análisis para identificar y delimitar las áreas rurales debería ser la local, dado que de realizarlo a una escala mayor como la comarcal, provincial, o regional, se correría el riesgo de perder las particularidades y singularidades de estos espacios. Estos autores propusieron un sencillo procedimiento para la jerarquización rural de los municipios en Galicia. El método está basado en la selección de 46 indicadores y la asignación de puntos a cada indicador con el objeto de la elaboración de un "ranking de ruralidad" que les permitió clasificar los municipios gallegos en función de su grado de ruralidad. De los resultados que obtuvie- 
ron diferenciaron cinco tipos de áreas rurales: los situados en las zonas muy urbanizadas, los de gran atractivo turístico, los que presentan actividades diversificadas, los que tienen un predominio agrícola, y los de difícil acceso o marginales.

Lois González (2014), llevó a cabo una tipificación simple de los distintos tipos de espacios rurales en Galicia adoptando tres criterios y estableciendo otros tantos niveles de ruralidad en este espacio: la ocupación humana del territorio medida en densidades de población, el peso de la actividad agraria en las estructuras económicas tanto locales como comarcales, y la distancia a las principales ciudades. Teniendo en cuenta estas tres variables el autor diferenció tres grados de ruralidad: áreas rurales con un efectivo humano aún destacado y que estarían influidas por la urbanización difusa de las periferias urbanas, áreas más alejadas y periféricas con un elevado grado de despoblación y envejecimiento $y$, por último, el rural que aún conserva un sustrato agrario significativo y que concentra a una buena parte de las principales comarcas productoras primarias de la región.

Paül Carril (2014), también para Galicia, utilizó siete indicadores y estableció cuatro intervalos en función de un determinado límite en los indicadores propuestos. El autor asigna distintos grados de ruralidad dependiendo del número de variables cumplidas. De este modo, considera municipios de elevada ruralidad los que cumplen seis o siete de los indicadores, y de ruralidad intermedia los que cumplen de dos a seis. La principal conclusión a la que llega el autor es que, en Galicia no existe un continuo urbano-rural, sino que el paso de lo urbano a lo rural es claro y tajante.

\section{LA NECESIDAD DE UN ÍNDICE DE RURALIDAD PARA GALICIA}

Las áreas rurales, en su proceso de transición al modelo postproductivista, se están adaptando a una nueva situación ambiental, demográfica, social, económica, territorial y política (Rubio Terrado, 1999). Una de las principales características de este modelo productivo es la diversificación de las actividades productivas, fenómeno al que se le acunó el nombre de pluriactividad, y que según Rubio Terrado (2010), debe ser objeto básico de las políticas rurales. En este sentido Van der Ploeg et al. (2000), señalan que la pluriactividad está siendo vista como una parte integral del desarrollo rural y que, combinada con la agricultura, puede llegar a ser una estrategia de subsistencia para las familias rurales. Autores como Kinsella, Wilson, Jong, y Renting (2000) apuntan que la pluriactividad no es algo nuevo y que ha sido una estrategia tradicional de adaptación económica de los problemas rurales para lograr la supervivencia cuando las condiciones del mercado se hacían duras. Pero para que la diversificación tenga éxito deben estar fundamentados en el profesionalismo y en la innovación (Bonnamour, 2001).

La obligación de delimitar las áreas rurales, sobre todo para orientar las líneas de actuación de las políticas de desarrollo rural, llevó a instituciones y organismos nacionales e internacionales a adoptar criterios propios para abordar esta compleja tarea utilizando, básicamente, los criterios de la densidad y volumen demográfico. La Unión Euro- 
pea, con el objeto de homogeneizar las estadísticas entre los estados miembros, creó definiciones estándares para los conceptos estadísticos, demográficos, geográficos y socioeconómicos utilizados en las diferentes encuestas sociales.

La Organización para la Cooperación y el Desarrollo Económico (OCDE) distinguió dos niveles jerárquicos de unidades territoriales, un nivel local y otro regional. A nivel local fueron consideradas áreas rurales aquellas que poseían una densidad de población inferior a $150 \mathrm{hab} / \mathrm{km}^{2}$ mientras que, a nivel regional, se diferenciaron tres tipos en función de la población que residían en cada una de ellas. Consideró regiones predominantemente rurales a aquellas que el 50\% de su población residía en áreas rurales, regiones significativamente rurales a las que entre el $15 \%$ y el $50 \%$ vivía en comunidades rurales y, por último, las regiones predominantemente urbanas en las que menos del $15 \%$ de su población vivía en zonas rurales.

La Oficina de Estadística de la Unión Europea (EUROSTAT) clasificó las regiones europeas utilizando también el criterio de la densidad de población. De un lado identifica las regiones muy densamente pobladas, que albergan a municipios con una densidad de población superior a los $500 \mathrm{hab} / \mathrm{km}^{2}$. Del otro, considera regiones intermedias a aquellas con municipios con una densidad de población inferior a los $500 \mathrm{hab} / \mathrm{km}^{2}$ y superior a $100 \mathrm{hab} / \mathrm{km}^{2}$. Por último, identifica las regiones rurales a las que tienen municipios con una densidad demográfica inferior a los $100 \mathrm{hab} / \mathrm{km}^{2}$.

A nivel nacional, el Instituto Nacional de Estadística (INE) español utiliza el volumen demográfico en lugar de la densidad de población, para delimitar las áreas urbanas de las rurales. Considera municipios urbanos a aquellos que tienen un volumen demográfico superior a los 10000 habitantes, municipios semiurbanos a los que tengan entre 2000 y 10000 habitantes y que, a su vez, tengan al menos una entidad de población con más de 2000 habitantes. Por último, considera municipios rurales a los que tengan menos de 2000 habitantes y todos aquellos que, teniendo una población entre 2000 y 10000 habitantes, no tengan ningún núcleo de población que supere los 2000 habitantes.

Teniendo en cuenta la dificultad que conlleva definir y delimitar el medio rural, se decidió diseñar un índice de ruralidad que se aplicó a Galicia. Pese a que ya se han elaborado algunas propuestas de delimitación de lo "rural" en esta región, la creación de un nuevo índice viene motivada, fundamentalmente, por varios factores. En primer lugar, se intenta aportar una delimitación más precisa de las distintas ruralidades que existen en este espacio. En las propuestas de delimitación del medio rural para Galicia expuestas en la revisión bibliográfica, solo una de ellas diferencia tres tipos de ruralidad en Galicia (Lois González, 2014) pero sin la elaboración de índice alguno. En el índice creado por López Ratón y Santiago Pérez (2005), pese a tener en cuenta un considerable número de variables, no define grados de ruralidad ya que, de los tres intervalos resultantes de la aplicación del índice, dos hacen referencia a espacios urbanos (urbano y semiurbano), y el tercero engloba a todo el medio rural. La última de las propuestas analizadas de delimitación del medio rural en Galicia de Paül Carril (2014), no crea un índice propiamente dicho, sino que establece la delimitación de los grados de ruralidad (en este caso dos) en 
función de un determinado valor en las variables seleccionadas, llegando a la conclusión de que en esta región no existe un continuo rural urbano, sino que el paso de lo rural a lo urbano es tajante.

Otra de las razones por las que se decidió crear un nuevo índice de ruralidad en Galicia, vino motivada por la necesidad, desde nuestro punto de vista, de incorporar la distancia, medida en tiempo de desplazamiento a los principales centros urbanos, a la hora de establecer los distintos grados de ruralidad. Esta variable, junto con otros indicadores que se expondrán a continuación, combinadas de forma ponderada, resultó un índice que delimita no solo las áreas urbanas y rurales, sino que, diferenciando varios grados de ruralidad, ayuda a comprender mejor las distintas realidades del mundo rural.

Quizás, el aspecto que más influyó a la hora de proponer este índice de ruralidad, fue la necesidad de elaborar un índice que dibujase el panorama urbano rural en Galicia y que sirviese para analizar tanto la diversidad del medio rural en este territorio, como los procesos de urbanización que están teniendo lugar tanto en los municipios más cercanos a las ciudades, como a lo largo de todo el Eje Atlántico. La realidad económica y social de los municipios que han experimentado un fuerte crecimiento urbano por su proximidad a las ciudades, es muy distinto de aquellos núcleos que conforman las villas y cabeceras de comarca que se emplazan en un contexto mucho más rural. Se cubre, desde nuestro punto de vista, la necesidad de delimitar con una mayor precisión los diferentes grados de ruralidad en Galicia, ayudando a orientar y aplicar las políticas de desarrollo rural. Esta necesidad de delimitación se hacía cada día más patente debido a los profundos cambios que estos espacios han experimentado en los últimos años. Y esta heterogeneidad es, si cabe, más acusada en el caso de Galicia. Se trata de una región que fue eminentemente rural, con unas características propias donde las distancias, su situación periférica, y la dispersión de sus asentamientos condicionaron su desarrollo.

El índice de ruralidad que aquí se presenta se basa en una fórmula en la que se combinaron seis variables: densidad de población, evolución de la población en los últimos diez años, tasa de envejecimiento, número de viviendas por cada cien habitantes, población ocupada que trabaja en el sector agrario, y el tiempo de desplazamiento en minutos a la ciudad más próxima de 50000 habitantes o más. Los datos referidos a la población fueron consultados del Padrón municipal de habitantes del Instituto Nacional de Estadística español para el año 2013, los datos de variación poblacional en los diez últimos se refieren al período 2003-2013, y los datos de viviendas y la población ocupada en el sector primario se consultaron en el Censo de Población y Viviendas del año 2011 del Instituto Nacional de Estadística. Por último, el cálculo de las distancias, medida en minutos y desplazándose en coche, se llevó a cabo desde cada municipio a la ciudad más próxima de 50000 habitantes utilizando la aplicación Google Maps teniendo en cuenta la ruta más rápida.

Un considerable número de los núcleos rurales que están próximos a las ciudades experimentaron en las últimas décadas, un notable crecimiento demográfico y económico al absorber población urbana en busca de una mayor calidad de vida, viviendas más baratas y al mismo tiempo con una buena oferta de servicios. La distancia se midió en tiempo ya que una buena red de comunicaciones, puede hacer que espacios rurales más 
alejados, pero bien comunicados, experimenten una dinámica económica y demográfica más positiva que otros más cerca físicamente, pero a una distancia en tiempo mayor. La fórmula que se utilizó para crear el índice de ruralidad es la que sigue:

$$
I r=\frac{\log (\text { dpob })^{-1}+\log (\text { evpob })^{-1}+\log (\text { tenv })+\log (v i v h a b)+\log (\text { pobagr })+\log (\text { tdesp })}{n}
$$

dpob: Densidad de población.

evpob: Evolución de la población en los últimos diez años.

tenv: Tasa de envejecimiento.

vivhab: Número de viviendas por cada 100 habitantes.

pobagr: Población ocupada que trabaja en el sector agrario.

tdesp: Tiempo de desplazamiento a la ciudad más próxima de 50000 habitantes o más.

$n$ : Número de variables.

Una vez calculado el índice para los 315 municipios gallegos ${ }^{\mathrm{ii}}$, se procedió a normalizar los valores entre -1 y 1 , siendo los valores más próximos a 1 los municipios con un mayor grado de ruralidad y los municipios con valores próximos a -1 los más urbanos. Con el fin de llevar a cabo, además de una clasificación urbano-rural, se decidió establecer tres grados de ruralidad y otros dos para clasificar los municipios urbanos. El resultado de la normalización del índice de ruralidad y su clasificación para Galicia fue el siguiente: 7 municipios urbanos (2\%), 79 semiurbanos (25\%), 82 semirurales (26\%), 106 rurales (34\%), y 41 muy rurales (13\%) (fig. 1).

El resultado de la delimitación urbano rural tras la aplicación del índice de ruralidad, evidencia el fuerte componente rural de este territorio y resalta su diversidad a través de los grados de ruralidad definidos. La utilización del criterio de la densidad de población en las propuestas de EUROSTAT y la OCDE en Galicia, dibujan un panorama eminentemente rural, además de limitarse a una simple clasificación de municipios urbanos y rurales, con lo que no se refleja la diversidad del mundo rural. En el caso del INE, pese a usar el volumen demográfico en lugar de la densidad de población, adolece de la misma debilidad aún introduciendo una categoría más. La ventaja del índice de ruralidad con respecto al propuesto por el INE, EUROSTAT y la OCDE, radica en que, además de tener en cuenta indicadores demográficos, incorpora otros relacionados con la actividad económica, la vivienda y la distancia a las siete ciudades de la comunidad.

Esta delimitación permite distinguir una ruralidad intensa, que se corresponde con la montaña de Lugo y Ourense además de algunos municipios emplazados en el interior de las provincias de Pontevedra y A Coruña, afectados por un intenso proceso de despoblación y declive económico; una ruralidad moderada que abarca la mayor parte de las provincias de Lugo y Ourense, salvo sus dos capitales y coronas urbanas y que engloba a municipios que no están afectados por el abandono ni por el crecimiento de las ciudades y sus periferias y donde domina un tejido agrario y ganadero importante. Por último, los municipios con un grado de ruralidad débil que son aquellos que están próximos a los núcleos urbanos o bien conectados con ellos, y los que albergan a las villas y cabeceras de 
comarca. Junto a ello, el índice también permite diferenciar a la Galicia urbana y semiurbana que conforma el Eje Atlántico, un área que se articula en torno a la autopista AP-9 y que se extiende de norte a sur desde Ferrol a Vigo-Tui (fig. 1).

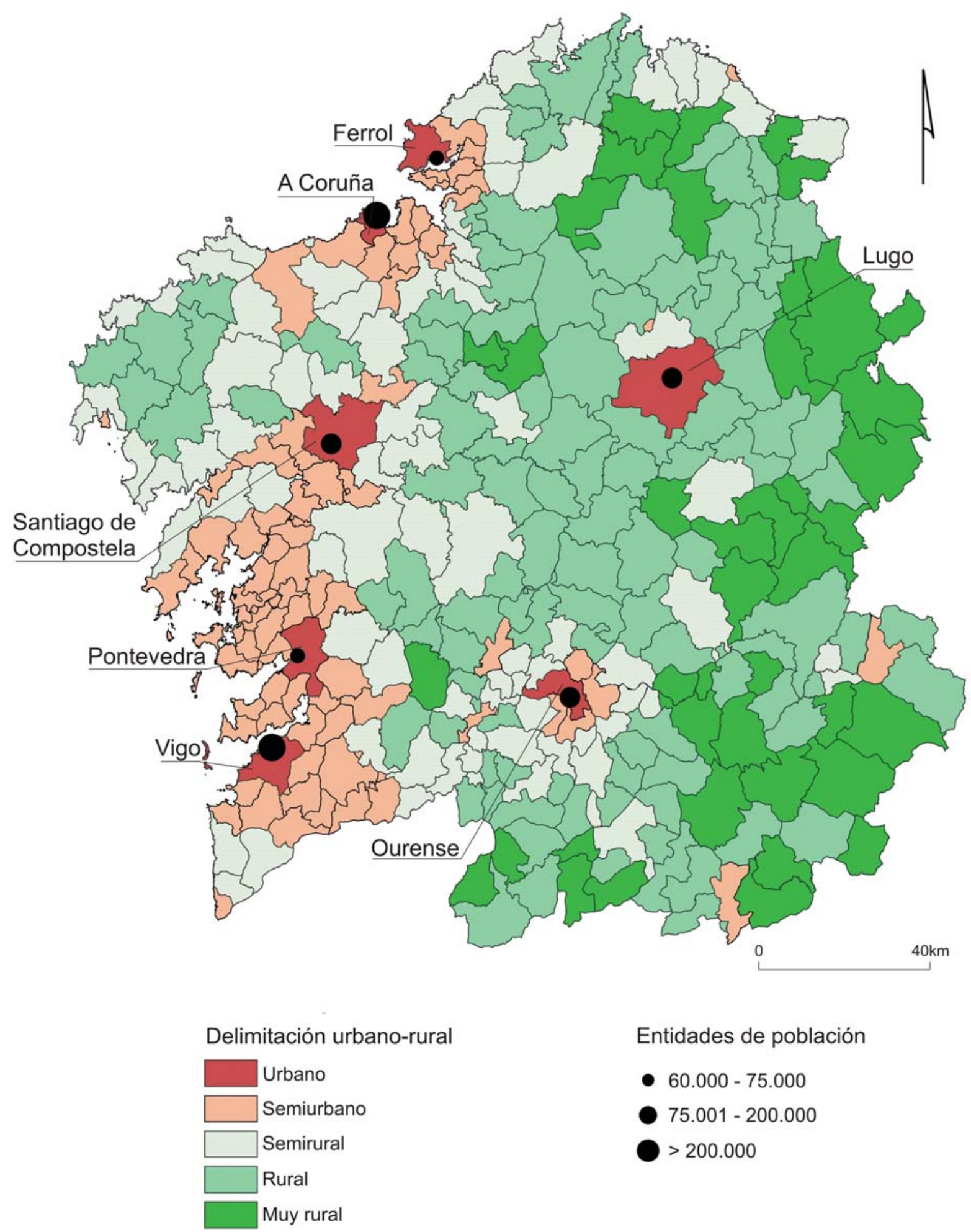

Fig. 1 - Índice de ruralidad. Delimitación urbano-rural en Galicia. Figura en color disponible en línea.

Fig. 1 - Index of rurality. Rural-urban delimitation in Galicia. Colour figure available online. 
El índice de ruralidad elaborado para el caso de Galicia representa, desde nuestro punto de vista, los diferentes grados de ruralidad en esta comunidad y podría ser muy útil a la hora de aplicar las políticas de desarrollo rural, en especial el Programa de Desarrollo Rural para el período 2014-2020. De acuerdo con la estrategia Europa 2020, uno de los objetivos estratégicos de la política de desarrollo rural del período 2014-2020 es lograr un desarrollo territorial equilibrado de las economías y comunidades rurales, incluyendo la creación y conservación del empleo. Pero para ello es necesario, previamente, delimitar y diferenciar las distintas realidades en estos espacios para ajustar y diseñar actuaciones en la línea de conseguir los objetivos propuestos. El índice de ruralidad que aquí se presenta, puede ser también útil a la hora de aplicar las distintas iniciativas de la Estrategia Gallega de Especialización Inteligente (RIS3 Galicia) destinadas a transformar el modelo productivo gallego hacia uno nuevo basado en la innovación y el conocimiento.

\section{A MODO DE CONCLUSIÓN}

Fueron muchos los intentos y esfuerzos llevados a cabo para definir y delimitar las áreas rurales y, pese a ello, a día de hoy no existe una definición comúnmente aceptada entre la comunidad científica, lo que evidencia la complejidad de este proceso. La mayor parte de las delimitaciones se asientan en criterios demográficos, como los usados por instituciones y organismos públicos, pero es necesario diseñar indicadores que tengan en cuenta aspectos sociales, culturales y económicos que permitan identificar los espacios rurales de una forma más objetiva y comparable.

La necesidad, para el caso de Galicia, es si cabe más apremiante debido a las intensas transformaciones que han tenido lugar en su medio rural en las últimas décadas. Se ha pasado de un espacio eminentemente rural y agrícola, donde tres de cada cuatro habitantes residían y trabajaban en el campo, a convertirse en una sociedad eminentemente urbana y terciarizada. El fuerte proceso urbanizador que ha tenido lugar en esta región en las últimas décadas, así como el fuerte declive económico y demográfico en el que están sumergidos los municipios rurales más periféricos, dio como resultado un amplio elenco de "ruralidades" que van desde los tradicionales espacios agrarios "modernizados", a espacios residenciales con un peso mayoritario del sector servicios que mermaron su pasado vínculo rural.

El índice de ruralidad propuesto permite dibujar la realidad urbano-rural en Galicia. Además de conocer la diversidad de su medio rural estableciendo tres gradientes de ruralidad, muestra los procesos de urbanización que están teniendo lugar en las áreas más cercanas a los centros urbanos y a lo largo del Eje Atlántico. La diferenciación de las distintas realidades que conviven en este territorio, permitirán a los gestores públicos orientar de un modo más preciso, las estrategias de desarrollo. Tras aplicar el índice de ruralidad, forman parte de la Galicia urbana siete municipios clasificados como urbanos (2\%), que se corresponden con las siete ciudades gallegas y 79 municipios semiurbanos (25\%) donde los procesos de urbanización son más intensos y sus características, otrora rurales, están muy mermadas debido a la fuerte influencia de las ciudades. 
De otro lado, la Galicia rural la forman ochenta y dos municipios semirurales (26\%), ciento seis rurales (34\%), y cuarenta y uno (13\%) muy rurales. Este último grupo lo componen los municipios más periféricos y con unas características físicas que impidieron que el sector agrario tradicional se transformase y modernizase, y su distancia a las ciudades condicionase el desarrollo de un tejido económico alternativo al sector primario. En el otro extremo del panorama rural gallego están los municipios semirurales, áreas rurales dinámicas que acogen población procedente de las ciudades y que se benefician de su proximidad. Sus "nuevos" pobladores procedentes de la ciudad, buscan una mayor calidad de vida y motivados, a su vez, por un menor coste de la vivienda y una amplia dotación de servicios.

Por último, están los municipios categorizados como rurales, que no están afectados por la influencia del proceso urbanización que envuelve a las ciudades, ni tampoco sufren la fuerte pérdida demográfica, el envejecimiento y el declive económico de los más periféricos. Son espacios donde tiene mucha presencia el sector primario, especialmente la agricultura y la ganadería. En estos municipios, la agricultura sigue siendo el elemento que los define, aunque desprendidos de su carácter tradicional y de autoconsumo, para convertirse en una agricultura comercial.

El diseño y aplicación de este índice de ruralidad permite, no solo acercase de un modo más preciso, a "resolver" el problema de la delimitación urbano-rural en Galicia, sino que contribuye a conocer mejor la diversidad y heterogeneidad de estos espacios de cara al diseño de estrategias y programas de desarrollo rural.

\section{REFERENCIAS BIBLIOGRÁFICAS}

Armesto López, X. A. (2005). Notas teóricas en torno al concepto de postproductivismo agrario. [Theoretical notes on the concept of agricultural post-productivism]. Investigaciones Geográficas, 36, 137-156.

Ballas, D., Kalogeresis, T., \& Labrianidis, L. (2003 August). A comparative study of typologies for rural areas in Europe. In ERSA Conference Papers, European Regional Science Association. Jyväskylä: Finland.

Bericat Alastuey, E. (1993). La teoría del vacío rural [The rural vacuum theory]. In Consejería de Agricultura y Pesca, Junta de Andalucía (Eds), El desarrollo rural a las puertas del siglo XXI [Rural development at the gates of the 21st century] (pp. 45-54). Sevilla: Junta de Andalucía.

Bibby, P., \& Shepherd, J. (2004). Developing a new classification of urban and rural areas for policy purposes. the methodology. London: Department for Environment, Food and Rural Affairs.
Bonnamour, J. (2001). El mundo rural ante el nuevo siglo: retos y desafíos [The rural world facing the new century: issues and challenges]. In F. García Pascual (Ed.), El mundo rural en la era de la globalización: incertidumbres y potencialiades [The rural world in the era of globalization: uncertainties and potentialities] (pp. 23-41). Madrid: Ministerio de Agricultura, Pesca y Alimentación.

Bosak, J., \& Perlman, B. (1982). A review of the definition of rural. Journal of Rural Community Psychology, 3(1), 3-34.

Bouhier, A. (1979). La Galice. Essai d'interpretation d'un vieux complexe agraire [Galicia. Interpretation of an old agrarian complex]. La Roche-sur-Yon (Vendée) : [s.n.]. (Imp. Yonnaise).

Camarero, L. (1993). Del éxodo rural y del éxodo urbano [Rural exodus and urban exodus] Madrid: Ministerio de Agricultura, Pesca y Alimentación.

Ceña-Delgado, F. (1995). Planteamientos económicos del desarrollo rural: una perspectiva histórica 
[Economic approaches to rural development: a historical perspective]. In E. Ramos, \& J. Cruz (Coords.), Hacia un nuevo sistema rural [Towards a new rural system] (pp. 91-129). Madrid: Ministerio de Agricultura, Pesca y Alimentación.

Cloke, P. (2006). Conceptualizing rurality. In P. Cloke, T. Marsden, \& P. Mooney (Eds.), The handbook of rural studies (pp. 18-28). London: SAGE Publications.

Cloke, P. (1977). An index of rurality for England and Wales. Regional Studies, 11, 31-46.

Cloke, P., \& Goodwin, M. (1993). Rural change: structured coherence or unstructured incoherence? Terra, 105(3), 166-174.

Clout, H. (1993). European experience of rural development. London: Rural Development Commission.

De Arriba, R. (2011). Mutaciones en la Europa rural y límites de la política económica de desarrollo rural en la actualidad [Mutations in rural Europe and limitations of the economic policy of rural development at present]. Cuadernos de desarrollo rural, 8(66), 177-194.

Entrena-Durán, F. (2012). La ruralidad en España: de la mitificación conservadora al neorrularismo [Rurality in Spain: From conservative mythification to de-urbanisation]. Cuadernos de desarrollo rural, 9(69), 39-65.

Esparcia, J. (2012). Evolución reciente, situación actual y perspectivas futuras en el desarrollo rural en España y en la UE [Recent evolution, current situation and future perspectives in the rural development in Spain and the EU]. Revue Marocaine d'Administration Locale et de Développement, 79, 53-84.

Friedland, W. H. (2002). Agriculture and rurality. Beginnig the final separation? Rural Sociology, 67(3), 350-371.

García de León, M. (1996). La ciudad y el campo: las imágenes opuestas de "el otro" [The city and the countryside: the contrasting images of "the other"]. In Ministerio de Agricultura (Eds.), El campo y la ciudad [The countryside and the city] (pp. 13-44). Madrid: Ministerio de Agricultura.

García Fernández, J. (1975). Organización del espacio y economía rural en la España Atlántica [Organisation of space and rural economy in Atlantic Spain]. Madrid: Ed. Siglo XXI.
García Ramón, M. D., Tulla i Pujol, A. F., \& Valdovinos Perdices, N. (1995). Geografía rural [Rural geography]. Madrid: Síntesis.

García Sanz, B. (1998). La sociedad rural ante el siglo XXI [The rural society facing the 21 st century]. Madrid: Ministerio de Agricultura, Pesca y Alimentación.

Guibertau Cabanillas, A. (2002). Fortalezas y debilidades del modelo de desarrollo rural por los agentes locales [Strengths and weaknesses of the rural development model by local stakeholders]. In Márquez Fernández (Coord), Nuevos horizontes en el desarrollo rural [New horizons in rural development] (pp. 87-104). Madrid: Akal.

Halfacree, K. (2007). Sill surprises in store. Revisiting the ordinary in rural geography. Documents d’Anàlisi Geogràfica, 50, 87-103.

Halfacree, K. (1995). Talking about rurality: social representations of the rural as expressed by residents of six English parishes. Journal of Rural Studies, 11(1), 1-20.

Halfacree, K. (1993). Locality and social representation: space, discourse and alternative definitions of the rural. Journal of Rural Studies, 9(1), 23-27.

Hoggart, K. (1990). Let's do away with rural. Journal of Rural Studies, 6(3), 245-257.

Kinsella, J., Wilson, S., Jong, F., \& Renting, H. (2000). Pluriactivity as a livelihood strategy in Irish farm households and its role in rural development. Sociologia Ruralis, 40(40), 481-496. doi: 10.1111/1467-9523.00162

Lawrence, M. (1996). Reading, writing, and the rural. Journal of Rural Studies, 12(1), 79-85.

Lázaro Araujo, L. (1995). El desarrollo rural en el contexto de la Unión Europea [Rural development in the context of the European Union]. In E. Ramos, \& J. Cruz (Coords.), Hacia un nuevo sistema rural [Towards a new rural system] (pp. 237-255). Madrid: Ministerio de Agricultura, Pesca y Alimentación.

Lois González, R. C. (2014). As transformacións recentes nun rural galego que mudou [Recent transformations in a changed rural Galicia]. In P. Saavedra Fernández, \& R. Rodríguez González (Dir.), A terra en Galicia: pasado presente e futuro [A land in Galicia: present past and future] (pp. 112-137). Granada: Universidade Internacional Menéndez Pelayo y Lalín, Concello de Lalín. 
López González, F. J., Crecente Maseda, R., \& Miranda Barros, D. (2004). Caracterización rural de los municipios gallegos de cara a una acertada aplicación de iniciativas comunitarias de desarrollo rural [Rural characterisation of the Galician municipalities in terms of a successful application of community initiatives in rural development]. Bilbao: Congreso Internacional de Ingeniería de Proyectos.

López Ratón, M., \& Santiago Pérez, M. I. (2005). Construcción de un índice de ruralidad y clasificación de los municipios gallegos [Creation of a rurality and classification index of Galician municipalities]. Guimarães: I Congresso de Estatística e Investigação Operacional da Galiza e Norte de Portugal.

Marsden, T., Murdoch, J., Lowe, P., Munton, R., \& Flynn, A. (1993). Constructing the countryside. London: University College London Press.

Márquez Fernández, D. (Coord.). (2002). Nuevos horizontes en el desarrollo rural [New horizons in rural development]. Madrid: Akal.

Muilo, T. (2010). Needs for rural research in the northern Finland context. Journal of Rural Studies, 26(1), 73-80.

Organisation de Cooperation et de Developpement Economiques (OCDE) (1994). Créer des indicateurs ruraux pour étayer la politique territoriale [Create rural indicators to support territorial policy]. París: OECD.

Paül Carril, V. (2014). Que espazos rurais nun país urbano? Dúas aproximacións xeográficas ao rural galego [What rural spaces are there in an urban country? Two geographical approaches to rural Galicia]. In R. Lois, \& D. Pino (Coords.), A Galicia Urbana [The Urban Galicia] (pp. 699-721). Vigo: Edicións Xerais de Galicia.

Prados, M. J. (2009a). Naturbanization: New identities and processes for rural-natural areas. In M. J. Prados (Ed.), Naturbanization: new identities and processes for rural-natural areas (pp. 3-7). London: Taylor \& Francis Group.

Prados, M. J. (2009b). Conceptual and methodological framework of naturbanization. In M. J. Prados, (Ed.), Naturbanization: new identities and processes for rural-natural areas (pp. 11-28). London: Taylor \& Francis Group.

Pratt, A. (1996). Discourses of rurality: loose talk or social struggle. Journal of Rural Studies, 12(1), 69-78.
Ramos, E., \& Romero, J. (1995). Para una concepción sistémica del desarrollo rural [For a systemic conception of rural development]. In E. Ramos, \& J. Cruz (Coords.), Hacia un nuevo sistema rural [Towards a new rural system] (pp. 49-89). Madrid: Ministerio de Agricultura, Pesca y Alimentación.

Roca, J. (2003). La delimitación de la ciudad: ¿una cuestión imposible? [City delimitation: an impossible question?] Ciudad y Territorio. Estudios Territoriales, 35(135), 17-36.

Rodríguez, A. (2011). Pertinencia y consecuencias de modificar los criterios para diferenciar lo urbano de lo rural [Relevance and consequences of modifying criteria to differentiate between the urban and the rural] In M. Dirven, R. Echeverri Perico, C. Sabalain, A. Rodríguez, D. Candia Baeza, C. Peña, \& S. Faiguenbaum (2011), Hacia una nueva definición de "rural" con fines estadísticos en América Latina [Towards a new definition of "rural" for statistical purposes in Latin America] (pp. 31-42). Santiago de Chile: Comisión Económica para América Latina y el Caribe (CEPAL), Naciones Unidas.

Rubio Terrado, P. (2010). Modelización de los cambios y evolución reciente del sistema rural español [Modelling of changes and recent evolution of the Spanish rural system]. Boletín de la Asociación de Geógrafos Españoles, 54, 203-235.

Sabalain, C. (2011). El concepto de rural en los países de la región [The concept of rural in the countries in the region] In M. Dirven, R. Echeverri Perico, C. Sabalain, A. Rodríguez, D. Candia Baeza, C. Peña, \& S. Faiguenbaum (2011), Hacia una nueva definición de "rural" con fines estadísticos en América Latina [Towards a new definition of "rural" for statistical purposes in Latin America] (pp. 21-29). Santiago de Chile: Comisión Económica para América Latina y el Caribe (CEPAL), Naciones Unidas.

Sancho Comíns, J., \& Reinoso Moreno, D. (2012). La delimitación del ámbito rural: una cuestión clave en los programas de desarrollo rural [Rural delimitation: a key issue in rural development programmes]. Estudios Geográficos, 73(273), 599-624. doi:10.3989/egeogr.2012.i273

Sancho Hazak, R. (1997). Estructura demográfica y tipificación de los asentamientos y áreas rurales españolas [Demographic structure and settlement typification and Spanish rural areas]. In C. Gómez Benito, \& J. González Rodríguez (Eds.), 
Agricultura y sociedad en la España Contemporánea [Agriculture and society in Contemporary Spain] (pp. 173-224). Madrid: Ed. CIS.

Shucksmith, M., \& Chapman, P. (1998). Rural development and Social Exclusion. Sociologia Ruralis, 38(2), 225-242.

Van der Ploeg, J. D., Renting, H., Brunori, G., Knickel, K, Mannion, J., Marsden, T. ...Ventura, F. (2000).
Rural development: from practices and policies towards theory. Sociologia Ruralis, 40(4), 391-408 .

Villares Paz, R. (1982). La propiedad de la tierra en Galicia (1500-1936) [Land ownership in Galicia (1500-1936)]. Madrid: Ed. Siglo XXI.

Woods, M. (2011). Rural. Key ideas in geography. Abingdon: Routledge.

i Esta publicación fue realizada en el marco del proyecto "Los retos en el sistema metropolitano español ante la Estrategia Europea 2020 en un contexto de crisis. La consolidación de un observatorio urbano y metropolitano", financiado por el Ministerio de Ciencia y Tecnología (CSO2013-47833-C4-1-R).

ii En el momento de la creación del índice de ruralidad aún no se habían producido la fusión de los municipios Oza dos Ríos y Cesuras y de Cerdedo y Cotobade. Estas fusiones dieron lugar a dos nuevos municipios de nombre Oza-Cesuras y Cerdedo-Cotobade. Estas fusiones fueron aprobadas por la Xunta de Galicia a través del DECRETO 83/2013 del 6 junio (DOG Núm. 108, 7 de junio de 2013) y del DECRETO 134/2016 del 22 de septiembre (DOG Núm. 197, lunes 17 de octubre de 2016) pasando a tener la Comunidad Autónoma de Galicia desde este momento 313 municipios. 